\title{
High resolution PET, SPECT and projection imaging in small animals
}

\author{
M.V. Green*, J. Seidel, J.J. Vaquero, E. Jagoda, I. Lee, W.C. Eckelman
}

\begin{abstract}
Positron emission tomography, single photon emission computed tomography and planar projection imaging of radioactive tracers have long been in use for detecting and diagnosing disease in human subjects. More recently, advanced versions of these same technologies have begun to be used across the breadth of modern biomedical research to study non invasively small laboratory animals in a myriad of experimental settings. In this report, we describe some of the new instruments and techniques that make these measurements possible and illustrate, with a few examples, the potential power of these methods in modern biomedical research.
\end{abstract}

Keywords: Small animal imaging; Positron emission tomography; Single photon emission computed tomography; Planar; Mouse and rat models

\section{Introduction}

In vivo imaging of pharmaceuticals labeled with radionuclides has proven to be a powerful diagnostic tool in human subjects. Important applications of this technology can be found in oncology, neurology, cardiology and virtually every other medical discipline. Indeed, over the years, these same imaging methods have often been applied in small animals, but usually only within the nuclear medicine (NM) community, and usually only to evaluate the efficacy of new radiopharmaceuticals.

During the past 5 years or so, this situation has begun to change, not only in NM, but also across most of the other imaging modalities. In the case of NM, it has gradually been realized that with the right imaging equipment and radiopharmaceutical infrastructure, the entire NM armamentarium can be brought to bear in small animals to solve a host of experimental problems that emerge from contemporary biomedical research. Among the areas of greatest promise are applications in drug discovery and development [1], in the study of small animal models of human diseases, and in characterizing gene expression and phenotype changes that arise from genetic manipulations [2]. In order to carry out such studies successfully, however, imaging systems must be available that meet the demanding spatial resolution and sensitivity requirements imposed when imaging subjects thousands of times less massive than humans. Human NM scanners do not, in general, meet these requirements except under certain very specialized circumstances and so cannot be used for this purpose. Instead, a number of laboratories world-wide have embarked on the development of imaging systems tailored specifically to small animal imaging [3 13]. In many cases, this work has yielded devices with certain performance characteristics substantially better than commercial human scanners $[11,13]$, and has laid the foundation for the widespread use of NM techniques outside the NM community. In this report, we describe the general features of several of these devices and several applications that illustrate their use. First, however, we note some of the factors that distinguish small animal imaging studies from their human counterpart.

\section{The small animal imaging environment}

Mice and rats used in typical small animal studies weigh between 2030 and $200300 \mathrm{~g}$, respectively. The "standard man", on the other hand, weighs 70,000 g, several thousand times more than a mouse and several hundred times more than a rat. In round numbers, the linear dimensions of organs in these animals are 10 or more times smaller in each dimension than in human subjects. It follows, therefore, that if imaging studies in these animals are to be "equivalent" to human studies, the spatial resolution of an animal scanner must be about 10 (or more) times better than a human scanner. "Equivalent" in this context means that the animal organ is visualized on the animal scale with the same relative acuity as the human organ is imaged on the human scale. The first challenge, therefore, is to improve spatial resolution by a 
very large factor relative to human scanners $(>10)$, or in absolute terms, to a fraction of a millimeter.

The large difference in body mass between small animals and human subjects also introduces another potential complication: the effects of specific activity of the radiopharmaceutical (the amount of radioactivity per unit mass of drug). Contrary to intuition, high resolution imaging of small animals cannot be performed with tracer doses scaled downward in the ratio of animal to human body masses. Indeed, it can be shown that, to a first approximation, the same absolute amount of radioactivity must be used in both subjects. The reason for this is that the size of the resolution "voxel" appropriate to the mouse or rat scales with the mass of the animal. If the statistical precision of measurement of activity in that voxel is to be the same as in the equivalent human study, the same absolute amount of radioactivity must be delivered to the small animal voxel. This requirement, in turn, implies that the same absolute amount of drug must be given to the animal as to the human, since it is the drug that carries the radioactivity to that voxel. However, if a human dose is given to an animal, the concentration of drug in the animal may be thousands of time greater than in the human since the animal is thousands of times less massive. The possibility exists, therefore, that such large concentrations could, in some cases, cause pharmacological effects in small animals rather than act as tracers, e.g. when attempting to label low concentrations of receptors in the brain. It can be shown semi-quantitatively that this "specific activity" problem can be minimized by formulating radiopharmaceuticals with specific activities much higher than for human subjects and by increasing the sensitivity of the imaging device by a substantial factor. Fortunately, both of these avenues can be pursued in the context of small animal imaging.

Finally, a third set of issues must be considered when imaging small animals that are not often encountered when imaging human subjects: complications involving tracer administration, blood sampling, movement suppression and anesthesia, and maintenance of the animal during the imaging process.

All NM studies require administration of a tracer directed toward some particular target. Routes of administration include intravenous, intra-arterial, intra-peritoneal, intramuscular, oral, direct installation and inhalation. The size of these animals, however, presents significant challenges when the tracer must be administered directly into the circulation by vein or artery (often the most favored routes). Veins and arteries in mice and rats are small in diameter making such injections difficult. Similar difficulties are also encountered when blood samples must be drawn from veins or arteries for metabolite assays or for defining the tracer input function to an organ. In addition, the total volume of tracer injected or blood withdrawn must be kept small compared to the animal's blood volume to avoid compromising the animal's circulatory status.

Another important difference between human and small animal studies lies in the need to actively suppress move- ment of the animal during imaging. While most human subjects can remain relatively motionless, small animals must be restrained or placed under anesthesia. Both of these strategies potentially interfere with studies in which the time course of radioactivity through various organ systems is to be studied. Anesthetics often alter heart rate and respiration and can, in some cases, directly interfere with tracer uptake. Restraint without anesthesia can also alter the animal's metabolic status. This combination of factors can thus potentially distort the true time variation of tracer uptake that might be observed if the animal were in its natural state. Moreover, given the small size of structures being observed at high resolution, normal respiratory and cardiac movements could cause blurring of the acquired image data. These sub-millimeter/millimeter scale movements might, therefore, also have to be artificially controlled or otherwise countered by cardiac and respiratory gating.

Small animals also differ from human subjects in the level of maintenance required during an imaging study. For example, small animals possess a much larger body surface area to body mass ratio than human subjects and so lose heat much more readily. As a result, the animal's body temperature must be monitored and maintained artificially during extended imaging studies. Circulating warm water blankets, circulating warm air, heat lamps and other schemes have all been used for this purpose. In addition to monitoring body temperature, it has become increasingly common to record the animal's ECG signal, control respiratory rate through mechanical ventilation, measure and record blood gas levels and other important physiological variables. Such record keeping and control of the animal's metabolic state can significantly reduce inter-animal variability and greatly aid in the interpretation of the acquired image data.

As suggested above, substantial differences exist between imaging studies in humans and small animals that must be addressed if small animal studies are to be carried out successfully. These differences present, in many cases, significant challenges in their own right and not all of the problems alluded to above have been solved. Since many of these same problems arise in other imaging modalities, e.g. MR, CT, it may be that solutions found in one area can be directly adapted to the NM environment and vice versa.

Assuming that an animal can be properly prepared and maintained, the animal must be imaged by a device with sufficient spatial resolution and sensitivity to accomplish the prescribed imaging task. In the remainder of this report, we examine some of the factors that affect spatial resolution and sensitivity of PET, SPECT and planar imaging systems now in use to image small animals.

\section{PET imaging}

Resolution and sensitivity in small animal PET are limited by three important factors: (1) positron range; (2) 
non-collinear annihilation; and (3) the intrinsic spatial resolution and sensitivity of the imaging system.

Positron emission tomography (PET) relies on coincidence detection of paired $511 \mathrm{keV}$ photons generated when an emitted positron and a nearby electron totally annihilate one another. Spatial resolution in PET is influenced by this process since the positron travels some distance away from the disintegrating nucleus before encountering the electron. This distance, the "positron range", represents a built-in "blur" present in all PET images that depends on the kinetic energy imparted to the positron during decay. The higher this "endpoint" energy, the farther the positron will generally travel and the worse the blur. While this effect is usually small compared to other factors in human imaging, instruments with high intrinsic spatial resolution can be adversely affected depending on the positron-emitting isotope. Fluorine-18, for example, possesses a short positron range (fraction of a millimeter) that is small compared to the intrinsic resolution of the first generation of dedicated small animal scanners (about $12 \mathrm{~mm},[6,7,10$ 12]). Images of compounds labeled with this isotope, e.g. 2-fluoro-2-deoxyglucose, are thus only marginally degraded by positron range. Other positron-emitters such as $\mathrm{O}-15, \mathrm{~N}-13$, Tc$94 \mathrm{~m}$ and I-124, however, possess higher endpoint energies and greater ranges and can give rise to measurable image degradation even in first generation machines. This problem will be of much greater significance in second generation, 1-mm scanners where the positron range of almost all commonly available positron-emitters will be comparable to the system resolution.

Another source of image blur arises from non-collinear annihilation events. In such cases, positron/electron annihilation does not give rise to two $511 \mathrm{keV}$ photons moving away from each other along a straight line, as is assumed, but rather along two lines at slight angles with respect to one another. Image blur associated with this effect increases as detector separation increases, but can potentially be minimized in small animal imaging systems by reducing detector separation to a distance commensurate with the size of the animal. Reducing detector separation also has the beneficial effect of increasing the geometric detection sensitivity of the imaging system. Unfortunately, there is a limit to this strategy. As detector separation decreases, the so-called "depth-of-interaction" (DOI) effect becomes important. This effect results in an increased uncertainty in the location of the endpoints of lines-of-response connecting detector pairs. This uncertainty, in turn, causes spatial resolution to degrade. In ring-type scanners, DOI manifests itself as an increasing reduction in spatial resolution for off-axis sources as ring diameter shrinks. In other kinds of devices, resolution is decreased for lines-of-response defined by annihilation rays that enter the detectors at increasingly oblique angles as detector separation is reduced. It follows, therefore, that if the effect of non-collinear annihilation is to be reduced, and geometric sensitivity increased, by bringing the detectors closer together, some scheme must be devised to counter the DOI effect. Given the other practical virtues of reduced detector separation, e.g. smaller number of detectors needed to surround the animal and lower cost, it is not surprising that a number of novel methods have been proposed to reduce the DOI error for small diameter imaging systems. These methods include the use of multilayer phoswich scintillation crystal arrays in which the layer of interaction is determined by differences in light decay time between the scintillator in each layer $[14,15]$, and by locating events along a crystal by measuring the light output from each end of the crystal [16]. It has been shown that these schemes, which yield relatively crude estimates of interaction depth along a crystal, can significantly reduce the DOI effect at small ring diameters and these, or other methods, will likely be used in at least some second generation machines. It is noteworthy that an effective DOI correction capability is already present in a non-scintillator based imaging system [11].

Although range and DOI effects are important sources of image degradation, the design of first generation, dedicated small animal PET scanners has been dominated by the need to significantly improve the intrinsic spatial resolution of small animal scanners relative to human scanners. Indeed, enormous ingenuity has been shown in the development of high-resolution instruments and it is not uncommon for the very newest technologies to be first demonstrated in animal systems. In particular, systems are now in operation [7,13] or being designed [17] that exploit the properties of a new class of high-speed, high light output lutetium-based scintillators. Lutetium oxyorthosilicate (LSO), for example, possesses a scintillation efficiency more than five times higher than bismuth germanate (BGO), a stopping power for $511 \mathrm{keV}$ radiation only slightly less than BGO and a light decay time shorter than BGO by more than a factor of eight. This combination of advantages over BGO, the scintillator in most human PET scanners, makes it possible to: (1) reduce the cross-sectional area of individual LSO crystals significantly, thereby improving intrinsic spatial resolution; (2) stop incident $511 \mathrm{keV}$ radiation with good efficiency; (3) allow much higher counting rates due to the short light pulse duration; and (4) improve random coincidence rejection.

Advanced position-sensitive phototube technology $[18,19]$ is also being used to identify the crystal-of-interaction in arrays of such crystals. These devices possess high speed, compact design and excellent light collection efficiency. The use of solid-state devices instead of phototubes to read out arrays of scintillation crystals has also been first demonstrated at the system level in a small animal scanner [6].

Alternatives to scintillator-based PET scanners have also been explored. The novel converter plate/gas amplification imaging system devised by Jeavons et al. [11], for example, produces extremely high resolution images [20] suggesting that alternative technologies may offer some advantages over conventional designs. All of these technological and 


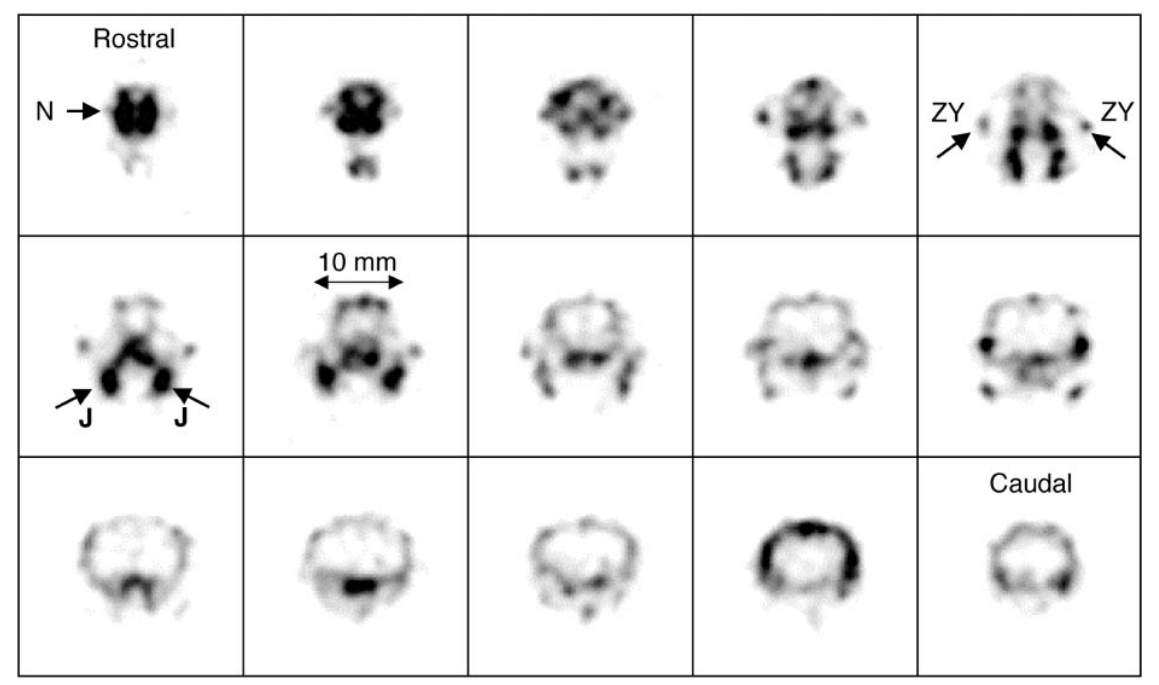

Fig. 1. $18 \mathrm{~F}$ fluoride coronal section images of the bones in the head of a mouse obtained with an experimental, bench top PET scanner. Images are 0.4 mm thick and spaced $1.2 \mathrm{~mm}$ apart. Spatial resolution in these images is slightly better than $1 \mathrm{~mm}$. Images were created using the $3 \mathrm{D}$ OSEM algorithm (30 iterations) containing a model of the system resolution response. $\mathrm{N}=$ nares, $\mathrm{ZY}=$ left, right zygomatic arches, $\mathrm{J}=$ left, right jawbones. Note image scale.

conceptual advances have emerged from a lively, vigorous and continuing research effort that promises even greater improvements in instrumentation in the future.

In addition to these purely instrument-related developments, it has also been recognized that spatial resolution can be significantly improved by using iterative reconstruction methods, rather than conventional filtered backprojection, to process image data acquired with any of these systems. These new algorithms provide a natural means of "de-blurring" the image data by incorporating a model of the physical performance of the imaging system and, potentially, positron range into the algorithm. Twenty to thirty percent improvements in spatial resolution have been demonstrated for these methods compared to filtered backprojection. Iterative methods, however, are extremely computationally intensive and exhibit subtle object-dependent convergence properties and it remains an important area of research to develop fast algorithms and to define cutoff criteria for properly terminating the iteration process. The importance of this effort cannot be understated since it is likely that the only way sub-millimeter resolution can be achieved is by combining very high intrinsic performance imaging systems with iterative algorithms that carefully model the physics of the entire imaging process. Interestingly, attainment of high resolution with these methods will not only require machines with high intrinsic spatial resolution, but machines with extremely high sensitivity if image noise is to be held low while allowing spatial resolution to increase with iteration number. While almost all firstgeneration machines utilize 3D data acquisition to improve sensitivity, the coincidence sensitivity per unit solid angle of detector differs appreciably. Ultimately, designs with the highest possible value of this parameter are most likely to achieve the sub-millimeter resolution goal.

An example of a study embodying many of these resolu- tion-enhancing advances is shown in Fig. 1. These images of the bones in the head of a mouse were made with an experimental, $1.3 \mathrm{~mm}$ intrinsic resolution bench-top scanner after labeling the animal's skeleton with 18-F fluoride. The scanner was designed specifically to achieve the highest possible spatial resolution but possesses a sensitivity too low for routine "production" imaging of small animals. In order to compensate for this low sensitivity, more than $2 \mathrm{~h}$ of data collection was required for the images shown in the figure. These image data were reconstructed using the iterative 3D OSEM algorithm containing a simple Gaussian model of the systems resolution response [21]. This imaging arrangement, therefore, simulates a high intrinsic spatial resolution scanner combined with iterative reconstruction and resolution recovery. Spatial resolution in these images is slightly better than $1 \mathrm{~mm}$ with a slice thickness of $400 \mu \mathrm{m}$. Such images may be obtained routinely by "production" scanners of the next generation.

Despite the various complicating factors noted in the preceding discussion, "production" small animal PET scanners now in use exhibit a spatial resolution in the $12 \mathrm{~mm}$ range $[6,7,1012]$, although with varying degrees of sensitivity. Although this resolution is poorer than the sub-millimeter goal noted earlier, an enormous variety of studies can still be carried out successfully in small animals. These studies include (usually) dynamic and static imaging of all the major body organs in the rat, including brain substructures. Brain imaging in mice with low contrast tracers such as 18-F 2-fluoro-2-deoxyglucose is limited at this resolution, but satisfactory studies of whole organs or large, widely separated brain structures, e.g. the striatum labeled with high affinity receptor ligands, can be obtained when the tracer biodistribution is favorable. PET is currently the most advanced tracer imaging technology for small animals and continues to be an area of intense research into all aspects of 
the imaging process, e.g. instrumentation, image reconstruction methods, etc.

\section{SPECT imaging}

A significant practical limitation to small animal PET imaging is the high cost of the infrastructure required to support this imaging modality. Short-lived PET radiopharmaceuticals must usually be created in an (expensive) on-site cyclotron/radiochemistry production facility. SPECT imaging, in contrast, can, in many cases, draw on a mature isotope distribution network and exploit the so-called "shake and bake" kits available to make a range of useful tracer compounds in-house. SPECT studies can also be carried out with commercially available scintillation cameras.

While SPECT possesses these desirable attributes, the small animal imaging options available with current technology are limited. The only current method for achieving SPECT spatial resolution in the $12 \mathrm{~mm}$ range is by using a pinhole collimator. With this device an image of the (small) object is projected through the pinhole onto a large area detector such as a conventional scintillation camera. The resulting magnification of the object improves the apparent resolution of the image depending on the size of the pinhole and other factors. Under normal circumstances, a full tomographic projection set can only be obtained by mechanically rotating the camera around the animal or by rotating the animal in front of the pinhole. In either case, some time is required to acquire a full data set so that true dynamic SPECT imaging is not possible, at least with this arrangement. In addition, the sensitivity of a single pinhole is necessarily low. Fortunately, when imaging small animals, the pinhole can be located close to the animal and sensitivity can often be comparable to other forms of single photon collimation. However, sensitivity in conventional single photon imaging cannot, in general, match that of PET since image formation is always by mechanical collimation, an inherently inefficient process. True dynamic SPECT imaging must await development of instruments specifically designed for this purpose. It should also be noted that reconstruction of pinhole SPECT image data requires special reconstruction algorithms that account for the pinhole projection geometry [22]. With such algorithms, spatial resolution in the reconstructed images is good near the central scanning plane but deteriorates away from the central plane due to inconsistent spatial sampling of the object.

Despite the limitations of existing instruments, high resolution SPECT images of a variety of "microsphere-like", or time-stationary, tracers can now be obtained, including those that permit visualization of regional cerebral blood flow, regional myocardial perfusion and regional bone metabolism to name just a few. As noted above, all of these studies can be carried out with only minor modifications to conventional, relatively inexpensive and available, gamma cameras. Examples of pinhole SPECT studies and a discussion of the advantages and disadvantages of SPECT pinhole imaging can be found in a recent review article by Weber and Ivanovic [23].

\section{Planar projection imaging}

If one is willing to give up the quantitative accuracy of tomographic imaging, single projection views of positron and single photon emitters can be obtained at high temporal resolution as well as high (2D) spatial resolution. Single photon imaging can be carried out with a stationary pinhole collimator viewing a stationary animal at a distance sufficient to visualize all or part of the animal. Dynamic imaging can be started at the moment of injection to yield a high temporal resolution image sequence of the passage of tracer through the body organs. The amount of single photon tracer that can be injected is subject to the same specific activity considerations noted earlier for PET radiopharmaceuticals. Time-activity curves can be created from regions-of-interest placed over different structures in the image sequence in order to quantify tracer transport. Although such studies collapse the third dimension onto a plane and thus introduce an uncertainty due to "background" activity in over and underlying structures, measures can be taken to minimize the magnitude of this error in small animals. Unlike human studies, calibration procedures can be devised in small animals by comparing in vivo count rates from body regions and organs to actually measured activities of these same structures counted in vitro. While such methods will always be approximate when applied to different animals, they can reduce background errors appreciably and in some cases, may produce results comparable to the equivalent tomographic study. It is also noteworthy that these kinds of studies do not require SPECT-capable instruments. A conventional, and relatively inexpensive, non-SPECT camera can perform this task (as well as small animal SPECT if the animal is rotated in front of the stationary camera).

While projection imaging of single photon tracers has been in use throughout NM for many years, projection imaging of positron emitters is relatively recent. If distributions of positron emitters are simply treated as photon sources, they too can be imaged through appropriately designed collimators [24]. However, the high energy of positron annihilation photons requires choices in collimator design that preclude use of this method in small animals.

Most of these difficulties can be overcome by performing projection imaging, not with collimators, but with positron emitters imaged with a pair of specially designed scintillation cameras in time coincidence. If these cameras have a relatively large field-of-view, and if the detectors possess high stopping power for $511 \mathrm{keV}$ radiation, then all or most of a small animal can be visualized with good sensitivity when placed between the detectors. If now only coincidence 

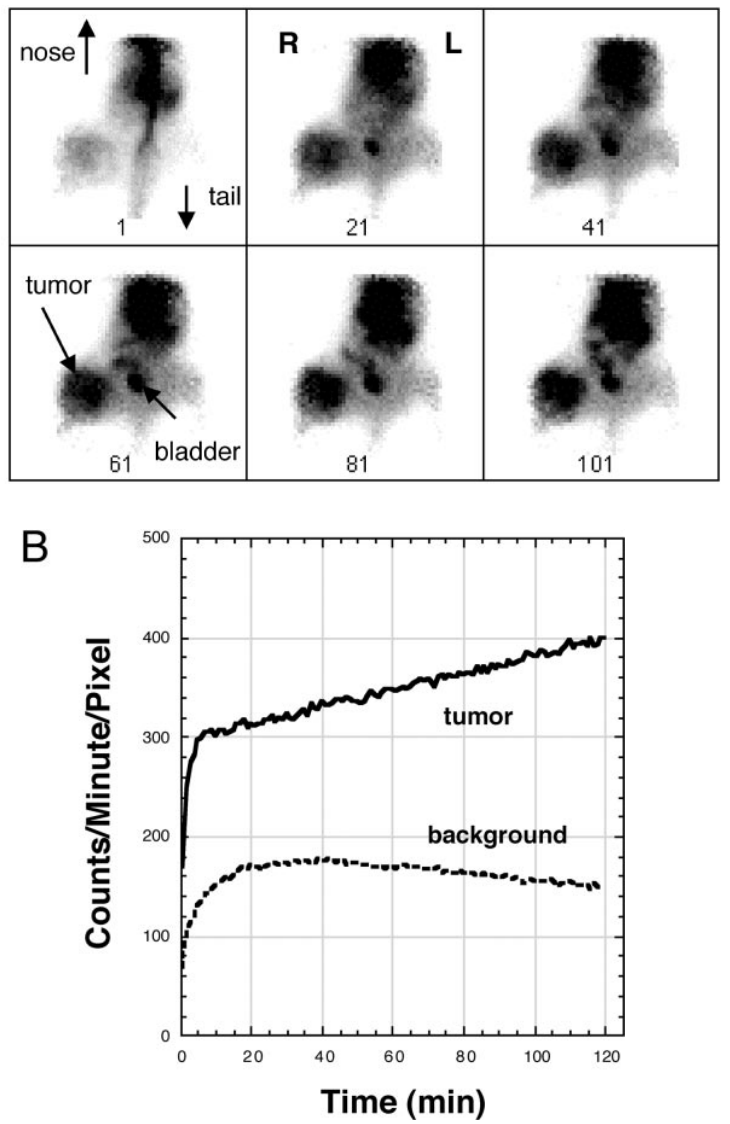

Fig. 2. (A) Six, 1 min duration anterior projection images of a tumor bear ing mouse spaced at $20 \mathrm{~min}$ intervals following intravenous injection of $18 \mathrm{~F}$ misonidazole, a tracer that accumulates in hypoxic cells. (B) Tumor time activity curve compared to a control region time activity curve from the opposite thigh. The tumor continues to accumulate activity throughout the observation period $(2 \mathrm{~h})$, while activity on the control side, after an initial increase, slowly declines. This behavior suggests that quantification of misonidazole activity in the tumor be delayed beyond $2 \mathrm{~h}$ until tumor activity becomes time stationary and background radioactivity is a small fraction of the tumor activity.

events that lie along lines nearly perpendicular to both detector faces are accepted, the activity distribution in the animal will be projected onto one of the detectors forming a planar image of the distribution. Such images, or image sequences, can be analyzed using the same strategies and calibration procedures noted above. This form of "collimator-less" planar projection imaging possesses the virtue, like its single photon cousin, that it can be carried out with relatively inexpensive equipment, yet yield a high (2D) spatial and temporal resolution result.

An example of this kind of positron projection imaging in a mouse is shown in Fig. 2. In order to obtain these images, the animal was placed between two BGO scintillation cameras in time coincidence (the "PiPET" [12], Projection Imager/PET). Each camera consists of an array of $22 \times 26$ individual BGO crystals, each $2 \mathrm{~mm} \times 2 \mathrm{~mm} \times 10 \mathrm{~mm}$ deep, coupled to a position-sensitive photomultiplier tube.
The effective field-of-view of each camera is about $45 \mathrm{~mm} \times 55 \mathrm{~mm}$, an area generally sufficient to image most of the body of a mouse or body sections of rats. The two scintillation cameras are rotated into a vertical orientation for projection imaging and the animal placed on a small table midway between the detectors. The animal can be injected while in the device and data acquisition started at the same time so that the complete time-course of tracer transit can be recorded.

The study portrayed in Fig. 2 was undertaken to determine the timing of the accumulation of F-18 labeled misonidazole in the hypoxic cells of a RIF tumor xenograft implanted in the animal's right thigh. The early images (2A) show the transport of tracer through the heart, lungs and liver while the later images show accumulation in the bladder and in the tumor. The time-activity curve from the tumor (2B) shows that $18-\mathrm{F}$ continues to accumulate in the tumor throughout the entire 2-h observation period. Activity on the tumor-free control side, however, eventually declines with time so that the ratio of tumor-to-tissue activity increases. Together, these data suggest that quantification of tumor activity should be delayed beyond $2 \mathrm{~h}$ until the tracer distribution becomes time-stationary. Such tracerspecific timing information is essential if imaging procedures are to be designed that will yield accurate and reproducible estimates of a tumor's response to various interventions.

\section{Conclusions}

Modern biomedical research offers numerous opportunities for the use of nuclear medicine in vivo imaging techniques to study small laboratory animals. PET, SPECT, planar imaging and variants of these techniques permit non-destructive assessment of functional changes in the same animal over time, a capability of great value when attempting to establish the efficacy of therapy or in establishing the functional consequences of a genetic manipulation in a rare and expensive animal. In response to these opportunities, advanced tracer imaging systems are being developed with the goal of allowing the whole range of human studies to be carried out in small animals with the same relative acuity as in man. At present, the various tracer imaging approaches in small animals are not equal in performance or cost, so that each modality occupies only a portion of the applications/cost spectrum. Moreover, no "production" imaging system now exists that operates in the submillimeter (or better) resolution range required to truly extend these methods to the smallest, and perhaps most important laboratory animal, the mouse. To alter this situation significantly will require the solution of complex and fundamental problems facing each of these modalities. Despite these limitations, existing methods now offer the biomedical scientist an unparalleled tool for studying the physiology and biochemistry of living systems non- 
destructively [25]. With continued effort, these methods will improve and very likely find even wider application.

\section{Summary}

Developments in modern biomedical research have made small laboratory animals, such as the mouse and rat, indispensable to the advancement of medical research. Tools that help the research scientist understand the biology, physiology or biochemistry of these animals under varying conditions in vivo are of potential importance in many areas, including phenotype characterization of genetically-altered animals, drug discovery and development and the assessment of therapies in animal models of human disease. In response to these opportunities, PET, SPECT and planar imaging systems have been developed that provide an overlapping range of functional capabilities in small animals. PET, the most technologically advanced method, can tomographically image regional static and dynamic tracer distributions in animals the size of rats and whole organs in the mouse. The next generation of "production" PET imaging systems will very likely exhibit millimeter (or better) spatial resolution that will extend this technology to smaller animals and improve performance in larger animals. Hybrid positron imaging systems, e.g. PiPET, that offer substantial functionality (high speed collimator-less positron projection imaging and rotational PET) at low cost may also play a role in introducing these methods to the research community.

SPECT and single photon projection imaging offer similar capabilities for tracers labeled with single photon emitters like Tc-99m. Although SPECT is currently restricted to imaging time-stationary "microsphere-like" tracers, many such compounds have been developed including those that visualize myocardial and cerebral blood flow and other important variables. Unlike PET, little research has been done to create specialized SPECT instruments for small animal imaging, though it is not difficult to imagine designs with sensitivities much higher than single pinhole imaging.

Despite their limitations, existing methods already allow a near endless variety of experiments to be carried out successfully in small animals provided care is taken to match the right instrument and technique to the right experiment. With continued technological refinements and a growing sophistication in applying these methods, small animal tracer imaging could, in the not too distant future, become a standard part of the biomedical research "tool box".

\section{References}

[1] Fowler JS, Volkow ND, Wang G J, Ding Y S, Dewey SL. PET and drug research and development. Journal of Nuclear Medicine 1999;40(7):1154 63.

[2] Gambhir SS, Barrio JR, Phelps ME, Iyer M, Namavari M, Satya murthy N, Wu L, Green LA, Bauer E, MacLaren DC, Nguyen K, Berk AJ, Cherry SR, Herschman HR. Imaging adenoviral directed reporter gene expression in living animals with positron emission tomography. Proceedings of the National Academy of Sciences 1999;96:2333 8.

[3] Watanabe M, Uchida H, Okada H, Shimizu K, Satoh N, Yoshikawa E, Ohmura T, Yamashita T, Tanaka E. A high resolution PET for animal studies. IEEE Transactions on Medical Imaging 1992;11(4):577 80.

[4] Strand SE, Ivanovic M, Erlandsson K, Franceschi D, Button T, Sjog ren K, Weber DA. Small animal imaging with pinhole single photon emission computed tomography. Cancer 1994;73(3):981 4.

[5] Lecomte R, Cadorette J, Rodrigue S, Lapointe D, Rouleau D, Bentourkia M, Yao R, Msaki P. Initial results from the Sherbrooke avalanche photodiode positron tomograph. IEEE Transactions on Nuclear Science 1996;43(3):1952 7

[6] Lecomte R, Cadorette J, Rodrigue S, Rouleau D, Yao R. Performance of the Sherbrooke avalanche photodiode PET scanner: a new high resolution device for animal research. Journal of Nuclear Medicine 1996;37(5):744.

[7] Cherry SR, Shao Y, Silverman RW, Meadors K, Siegel S, Chatziioan nou A, Young JW, Jones WF, Moyers JC, Newport D, Boutefnouchet A, Farquhar TH, Andreaco M, Paulus MJ, Binkley DM, Nutt R, Phelps ME. MicroPET a high resolution PET scanner for imaging small animals. IEEE Transactions on Nuclear Science 1997;44(3):1161 6.

[8] Ziegler SI, Fries O, Pichler B, Pimpl W, Roder F, Schwaiger M, Lorenz E. Prototype animal PET scanner with avalanche photodiode arrays and LSO crystals. Journal of Nuclear Medicine 1997;38(5): 160 .

[9] Fries O, Bradbury SM, Gebauer J, Holl I, Lorenz E, Renker D, Ziegler SI. A small animal PET prototype based on LSO crystals read out by avalanche photodiodes. Nuclear Instruments and Methods in Physics Research, Section A Accelerators Spectrometers Detectors and Associated Equipment 1997;387(1/2):220 4.

[10] Del Guerra A, Di Domenico G, Scandola M, Zavattini G. YAP PET: first results of a small animal Positron Emission Tomograph based on YAP:Ce finger crystals. IEEE Transactions on Nuclear Science 1998;45(6):3105 8.

[11] Jeavons AP, Chandler RA, Dettmar CAR. A 3D HIDAC PET camera with sub millimetre resolution for imaging small animals. IEEE Nuclear Science Symposium and Medical Imaging Conference. Toronto, Canada. Conference Record, IEEE CD ROM 07803 5024 3, 1998.

[12] Siegel S, Vaquero JJ, Aloj L, Seidel J, Gandler WR, Green MV. Initial results from a PET/Planar small animal imaging system. IEEE Trans actions on Nuclear Science 1999;46(3):571 5.

[13] Chatziioannou AF, Cherry SR, Shao Y, Silverman RW, Meadors K, Farquhar TH, Pedarsani M, Phelps ME. Performance evaluation of microPET: a high resolution lutetium oxyorthosilicate PET scanner for animal imaging. Journal of Nuclear Medicine 1999;40(7):1164 75 .

[14] Schmand M, Eriksson L, Casey ME, Andreaco MS, Melcher C, Wien hard K, Flugge G, Nutt R. Performance results of a new DOI detector block for a high resolution PET LSO research tomograph HRRT. IEEE Transactions on Nuclear Science 1998;45(6):3000 6.

[15] Seidel J, Vaquero JJ, Siegel S, Gandler WR, Green MV. Depth iden tification accuracy of a three layer phoswich PET detector module. IEEE Transactions on Nuclear Science 1999;46(3):485 90.

[16] Moses WW, Derenzo SE. Design studies for a PET detector module using a pin photodiode to measure depth of interaction. IEEE Trans actions on Nuclear Science 1994;41(4):1441 5.

[17] Fries O, Bradbury SM, Gebauer J, Holl I, Lorenz E, Renker D, Ziegler SI. A small animal PET prototype based on LSO crystals read out by avalanche photodiodes. Nuclear Instruments and Methods in Physics Research 1997;387(1/2):220 4.

[18] Seidel J, Siegel S, Gandler WR, Green MV. Accuracy of event posi tioning in a pixelated BGO array coupled to a miniature position sensitive phototube. Journal of Nuclear Medicine 1997;38(5):197P.

[19] Vaquero JJ, Seidel J, Siegel S, Gandler WR, Green MV. Performance 
characteristics of a compact position sensitive LSO detector module. IEEE Transactions on Medical Imaging 1998;17(6):967 78.

[20] Kendall L, Rayment D, Aigbirhio F, Baker HF, Chandler R, Clark J, Dettmar C, Dunnett SB, Jeavons A, Page K, Ridley R, Torres E, Turkheimer F, Visvikis D. In vivo PET analysis of the status of striatal allografts in the common marmoset. European Journal of Neuroscience 1998;10:371.

[21] Johnson CA, Seidel J, Carson RE, Gandler WR, Sofer A, Green MV, Daube Witherspoon ME. Evaluation of 3D reconstruction algorithms for a small animal PET camera. IEEE Transactions on Nuclear Science 1997;44(3):1303 8.

[22] Weber DA, Ivanovic M, Franceschi D, Strand SE, Erlandsson K, Franceschi M, Atkins HL, Coderre JA, Susskind H, Button T, Ljungg ren K. Pinhole Spect an approach to in vivo high resolution SPECT imaging in small laboratory animals. Journal of Nuclear Medicine 1994;35(2):342 8.

[23] Weber DA, Ivanovic M. Ultra high resolution imaging of small animals: implications for preclinical and research studies. Journal of Nuclear Cardiology 1999;6(3):332 44.

[24] Sloof GW, Visser FC, Bax JJ, van Lingen A, Eersels J, Knapp FF, Teule GJJ. Increased uptake of iodine 123 BMIPP in chronic ischemic heart disease: comparison with fluorine 18 FDG SPECT. Journal of Nuclear Medicine 1998;39(2):255 60.

[25] Tornai MP, Jaszczak RJ, Turkington TG, Coleman RE. Small animal PET: advent of a new era of PET research. Journal of Nuclear Medi cine 1999;40(7):1176 9.

Michael V. Green, MSc is currently Chief of the Imaging Physics Laboratory of the Nuclear Medicine Department, Clinical Center, National Institutes of Health (NIH) in Bethesda, Maryland, USA. His work at the NIH has included development of radiotracer methods for imaging cardiac function in exercising human subjects, oversight of the establishment of the imaging component of a large scale PET program and development of high performance radiotracer imaging systems for studying organ function in small animals. Mr Green is a co author on more than 225 papers, book chapters and abstracts.

Jurgen Seidel, $\mathrm{PhD}$ is a Staff Scientist in the Imaging Physics Labora tory, NIH. He obtained his PhD degree in Nuclear Physics from the University of Cologne in 1990 and joined the NIH shortly thereafter. As a Senior Investigator, Dr Seidel's activities have ranged from detector module design to image reconstruction methods to the mathematical modeling of novel imaging systems. He is presently engaged in bring ing an advanced small animal PET scanner into operation for use by NIH intramural scientists.
Juan J. Vaquero received his PhD degree (Doctor Ingeniero de Tele comunicación) from the Universidad Politécnica de Madrid in 1995 and joined the Imaging Physics Laboratory as a Visiting Fellow, NIH, in 1997. Dr Vaquero has worked both in industry and academia and is now engaged in the design of novel instruments for small animal imaging.

Elaine M. Jagoda holds an MS degree in Microbiology from George Washington University. She has worked in a research capacity in the pharmaceutical industry and in academia. She joined the PET Depart ment, NIH, in 1994 as a Research Microbiologist where she has specia lized in the development and characterization of new diagnostic radiopharmaceuticals.

Injae Lee received his $\mathrm{PhD}$ degree in Fiber Optics Materials Research from Rutgers University in 1998 and joined the Imaging Physics Laboratory, NIH, as a Visiting Fellow in 1999. Dr Lee has specialized in detector module design and fabrication and is currently engaged in the construction of an advanced small animal PET scanner.

William C. Eckelman, $\mathrm{PhD}$ is Chief of the Positron Emission Tomo graphy (PET) Department at the National Institutes of Health. His prin cipal focus is the design and synthesis of radiotracers capable of mapping biochemical pathways by external imaging. Dr Eckelman has been the Editor in Chief of Nuclear Medicine and Biology since 1985. He has received numerous professional awards including the Herman L. Blumgart Pioneer Lecture Award in 1985, the Paul C. Aebersold Award for Outstanding Achievement in Basic Science Applied to Nuclear Medicine in 1988 and the Georg deHevesy Nuclear Pioneer Award, Society of Nuclear Medicine in 1997. He has published over 250 research papers, books and book chapters. 